\title{
A hydrogen peroxide sensor for exhaled breath measurement
}

\author{
Dam T.V. Anh, W. Olthuis, P. Bergveld and A. van den Berg \\ The Lab-on-a-Chip group, MESA ${ }^{+}$Research Institute, University of Twente, \\ P.O. Box 217, 7500 AE, Enschede, The Netherlands. \\ E-mail: d.v.anh@el.utwente.nl, Url: http://www.bios.el.utwente.nl
}

\begin{abstract}
Summary: An increase in produced hydrogen peroxide concentration in exhaled breath (EB) of patients, who suffer from some diseases related to lung function, has been observed and considered as a reliable indicator of lung diseases. In the EB of these patients, hydrogen peroxide is present in the vapour phase together with water, thus one of the approaches of monitoring hydrogen peroxide in the EB is to condense it and then to perform the hydrogen peroxide measurement in the condensate.

Earlier, a hydrogen peroxide sensor based on an Electrolyte Metal Oxide Semiconductor Field-Effect Transistor, ${ }^{E}$ MOSFET, has been investigated. The sensor shows the possibility to measure hydrogen peroxide at a concentration of micro-molar level. Due to its miniaturizability, the sensor is able to detect hydrogen peroxide in a small solution volume, especially suitable for monitoring of hydrogen peroxide in the EB. In this paper, a new setup for condensation hydrogen peroxide in the EB is introduced. The setup consists of a cooling part using a Peltier cooler for condensation of the exhaled breath air and the ${ }^{E} M O S F E T$ based hydrogen peroxide sensor. Preliminary results on the collection and measurement of hydrogen peroxide in artificial EB are given.
\end{abstract}

Keywords: hydrogen peroxide sensor, ${ }^{E}$ MOSFET, exhaled breath

\section{Introduction}

Exhaled breath air consists of traces of many volatile compounds such as nitric oxide (NO) and carbon monoxide, and a water-vapour-saturated phase that contains aerosol particles with nonvolatile compounds such as isoprostane, $\mathrm{H}_{2} \mathrm{O}_{2}$, nitrite, chloride and proteins. These compounds reflect the composition of the bronchoalveolar extracellular lining fluid in the lung which is continuously subjected to many noxious agents presented in a polluted environment [1]. At healthy people, the concentrations of the non-volatile compounds in the exhaled breath are quite low [2], but it will dramatically increase at patients who suffer from certain diseases. When a person has lung inflammation, white blood cells (granulocyte type) release enzymes, hydrogen peroxide and other chemicals to kill the bacteria. An increase in the hydrogen peroxide concentration in the exhaled breath has been considered as a main and reliable indicator of lung diseases such as asthma, chronic obstructive pulmonary diseases $[3,4]$.

Therefore monitoring hydrogen peroxide level in the exhaled breath can provide reliable information for diagnostics and further treatment of mentioned diseases.

Up to now, a common method to collect the exhaled breath condensate, is to use a special cooling element including a freezing glass cooling tube and cooling machine, which has a refrigerator's circuit. Later, the obtained condensate is analyzed off-line by other techniques such as electrochemistry or spectroscopy due to the lack of a miniaturized hydrogen peroxide sensor.
In this paper, we present a setup, which allows to directly condensate the exhaled breath on a hydrogen peroxide sensor and then to perform the $\mathrm{H}_{2} \mathrm{O}_{2}$ measurement in the condensate. This setup consists of a cooling element using a Peltier cooler for condensation of the exhaled breath and an Electrolyte Metal Oxide Semiconductor FieldEffect Transistor, ${ }^{\mathrm{E}}$ MOSFET, based hydrogen peroxide sensor (see Fig. 1).

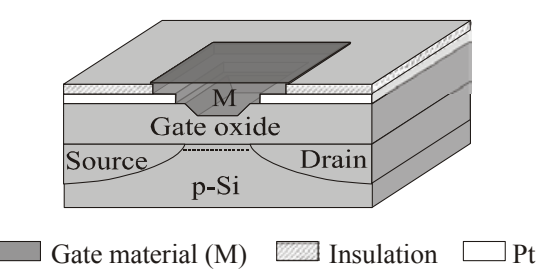

Fig. 1. The ${ }^{E}$ MOSFET based hydrogen peroxide sensor.

Earlier, the ${ }^{\mathrm{E}}$ MOSFET based sensor has shown the possibility to measure hydrogen peroxide at a concentration of micro-molar level [5]. Due to its miniaturizability, the sensor is able to detect hydrogen peroxide in a small volume of solution, especially suitable for monitoring of hydrogen peroxide in the exhaled breath.

\section{Experiments}

The cooling setup consists of a cooling part maintained by a Peltier element and a sensing part. When the humidified air containing hydrogen 
peroxide is blown towards the cooling part, the aerosol particles in the air are condensed into droplets containing hydrogen peroxide and collected at the sensing part. The sensing part consists of the ${ }^{\mathrm{E}}$ MOSFET based hydrogen peroxide sensor, which has an Os-polyvinylpyridine containing peroxidase (from BioAnalytical System) gate, and a counter electrode made from platinum. This electrode is used for applying an external nano-current between the gate electrode and the condensate during hydrogen peroxide measurement [4]. All chemicals used (Merk, Fluka) were of analytical reagent grade.

\section{Results and discussions}

Before measurement, a calibration curve of the sensor is performed. The response of the threshold voltage, $\mathrm{V}_{\mathrm{T}}$, of the ${ }^{\mathrm{E}}$ MOSFET to changes in a hydrogen peroxide concentration in solution is shown in Fig. 2 while small amounts of a stock hydrogen peroxide solution are added. Because the expected hydrogen peroxide concentration in medical applications is in micro molar range, an external reducing current of $10 \mathrm{nA}$ has been applied between the gate electrode and the platinum counter electrode to achieve a low detection limit of the sensor.

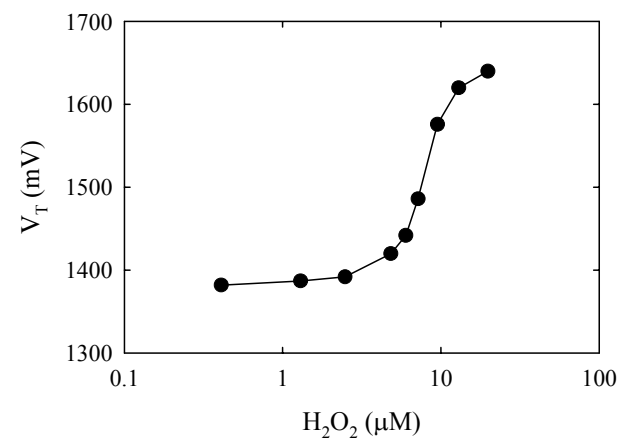

Fig. 2. Sensitivity of an Os-polyvinylpyridine containing peroxidase based sensor to hydrogen peroxide at the applied reducing current of $10 \mathrm{nA}$.

Following, monitoring of hydrogen peroxide in artificial exhaled breath air has been performed and preliminary results on the collection and measurement of hydrogen peroxide in artificial exhaled breath are given in Fig. 3. The hydrogen peroxide measurement in the condensate by using the ${ }^{\mathrm{E}}$ MOSFET based sensor has shown a result close to the expected value of hydrogen peroxide concentration in vapor phase.

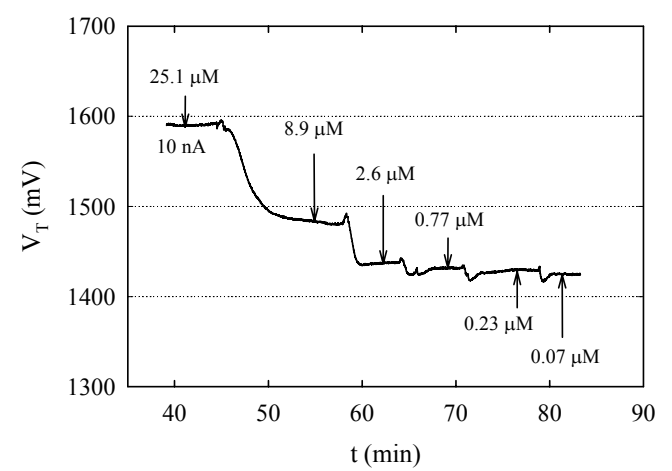

Fig. 3. Threshold voltage, $V_{T}$, of the ${ }^{E}$ MOSFET as a function of time when the $\mathrm{H}_{2} \mathrm{O}_{2}$ concentration in the condensate is decreased at the applied reducing current of $10 \mathrm{nA}$.

\section{Acknowledgements}

The authors would like to thank J. Bomer and P. Diepenbroek for technical support. The Dutch Technology Foundation (STW) is acknowledged for financial support.

\section{References}

1. J. Hunt, Journal of Allergy and Clinical Immunology, Vol. 110, 1 (2002) pp. 28 - 34.

2. http://www.jaerger-toennies.com

3. E.C. Lases, V.M. Duurkens, W. B. M. Gerritsen and F. J. L. M. Haas, Chest, Vol. 117, 4 (2000) pp. $999-1002$.

4. M. Zhou, Z. Diwu, N. Panchuk-Voloshina and P. Haugland, Analytical Biochemistry, Volume 253, 2 (1997) pp. 162 - 168.

5. Dam T. V. Anh, W. Olthuis and P. Bergveld, IEEE Sensors journal, Vol. 2, 1 (2002) pp. 2633. 